\title{
The concept of "harm" in Internet gaming disorder
}

\author{
DANIEL L. KING* and PAUL H. DELFABBRO \\ School of Psychology, The University of Adelaide, Adelaide, SA, Australia \\ (Received: February 12, 2018; accepted: March 16, 2018)
}

\begin{abstract}
Internet gaming disorder (IGD) is a proposed condition that refers to persistent gaming leading to clinically significant impairment. However, there have been few attempts to study the different types and degrees of harm caused by IGD. This commentary describes some of the negative intrapersonal and interpersonal effects of an extreme time investment in gaming activities in the context of IGD. Future research should examine the way in which IGD harms may occur at different levels and degrees. This may enhance the screening of individuals whose behavior is suspected to meet the definition of the proposed IGD criteria.
\end{abstract}

Keywords: Internet gaming disorder, harm, impairment, comorbidity, public health, negative effects

The proposed condition known as Internet gaming disorder (IGD) is thought to share many of its defining criteria with gambling and substance use disorders (American Psychiatric Association, 2013). At a behavioral level, engagement in excessive gaming can be associated with preoccupation, significant time spent away from other important activities, and a gradual intensification of activity. However, video gaming differs, for example, from gambling or consuming alcohol, in terms of how it is consumed and the potential consequences for players. For example, a problem gambler often experiences financial losses, which cause emotional distress and major life difficulties (e.g., conflict with a partner, loss of material assets, legal issues, bankruptcy, or loss of employment) (Langham et al., 2015). An alcoholic will experience similar interpersonal stressors and harms, in addition to negative effects on health and well-being, including heart disease, brain and liver damages, memory and attention problems, and the increased risk of physical injury while intoxicated, among many other related issues (World Health Organization [WHO], 2014). Such problems are rarely, if ever, reported by people affected by IGD.

An important question arises: How does IGD cause harm to players? Does this differ from other recognized addictive disorders? As mentioned, gamers can often, just like gamblers, spend much of their income on gaming activities. For example, some gamers may spend too much on gamingrelated purchases, including hardware and software (including digital content, such as "microtransactions"), thereby accruing debts on credit cards (particularly in the case of adolescents with access to parents' finances). Others may "chase losses" in the sense of spending money impulsively on monetization features in games to improve their playing performance (Soroush, Hancock, \& Bonns, 2014). However, it is thought that such financial expenditure by gamers is unlikely to ever rival that expended by problem gamblers. In support of this view, gamers will often highlight the relatively low costs of their gaming compared with other activities, after the initial purchase of gaming equipment. Indeed, gaming may be seen as inexpensive when costs are expressed as a function of expenditure divided by time spent playing (King, Kaptsis, Delfabbro, \& Gradisar, 2016).

Unlike substance misuse and addiction, the negative health effects of persistent gaming appear to be relatively mild. The most typically observed health-related "harms" of gaming include loss of weight due to restricted diet (or weight gain due to overeating), physical pain issues due to poor posture and repetitive strain injuries (Macgregor, 2000), and restricted and poor sleep and/or reverse sleepwake cycle that results in fatigue and lethargy (Männikkö, Billieux, \& Kääriäinen, 2015). In rare cases, gaming can produce photosensitive seizures (Chuang, 2006), but susceptible users may tend to avoid games that produce this reaction.

The primary way in which individuals with IGD are negatively affected by their gaming relates to the extreme time investment in gaming (i.e., 8-12 hr/day) (Baggio et al., 2016). Time spent gaming results in missed opportunities and the interference with, and displacement of, normal routine and functioning, including basic activities (i.e., sleep, eating, and personal hygiene), real-world social interaction (i.e., talking to people, meeting friends face-to-face, and visiting family), and important responsibilities (i.e., school, work, and care of pets and children). The recent ICD-11 description of gaming disorder emphasizes these notions (WHO, 2018). A gaming disorder markedly changes the user's priorities, which results in greatly diminished interest

\footnotetext{
* Corresponding author: Daniel L. King; School of Psychology, The University of Adelaide, Level 7, Hughes Building, Adelaide, SA 5005, Australia; Phone: +6188313 3740; Fax: +61 88303 3770; E-mail: daniel.king@adelaide.edu.au
}

This is an open-access article distributed under the terms of the Creative Commons Attribution-NonCommercial 4.0 International License, which permits unrestricted use, distribution, and reproduction in any medium for non-commercial purposes, provided the original author and source are credited, a link to the CC License is provided, and changes - if any - are indicated. 
in, and capacity to attend to, non-gaming-related information, people, and events. The affected individual becomes increasingly less able and less motivated to regulate gaming time. Consequently, the user neglects the real world and his/her roles within it. A wide range of negative outcomes follow this indifference and this may include increasing detachment from reality. A habitual pattern of gaming creates an expanding "void" in the person's life, where progression in important life areas, such as school or career, becomes anchored to the time at which gaming began. Over time, the individual may find it increasingly more difficult to resume their involvement in other activities, due to loss of self-efficacy and/or deterioration of skills. Such experiences appear to share some similarities with gambling, particularly on activities such as electronicgaming machines, where players can sometimes report entering into a "zone" (Schüll, 2012).

When not playing games, an individual with IGD is often preoccupied with gaming, consumed by thoughts of future gaming intentions and opportunities (King \& Delfabbro, 2016). The user may increasingly show less care about their present reality and attend less to non-gaming concerns. Preoccupation reduces the availability of cognitive resources for non-gaming tasks, which result in not learning at school, not completing work tasks efficiently or not completing them at all, and not interacting meaningfully with others. The lack of positive reinforcement and success in non-gaming areas further leads the user to retreat into gaming activities. Within a short period of time (i.e., less than 3 months), the user may experience negative consequences, such as failing at school, reprimands or lost productivity at work, and/or arguments with family or a partner. Negative mood states (i.e., usually irritability, sadness, and boredom) accompany the times when the individual is not playing or is less able to play (Dong, Wang, Du, \& Potenza, 2017; Kaptsis, King, Delfabbro, \& Gradisar, 2016). Such feelings may be amplified by other mood changes linked to poor diet and lack of sleep - which results in the user becoming more detached from the real world and seeking gaming for the relieving effects of play.

Thoughts of gaming for the user tend to be more automatic and require less mental effort and are more pleasant than thinking about the real-world matters. For some gamers, having to think about oneself and the quality of life outside of gaming may be very painful and lead to suicidal ideation (Wu, Lee, Liao, \& Chang, 2015). Such thoughts will often be "blocked out" by gaming-related behavior (e.g., browsing gaming websites). Individuals affected in this way can become less patient and respectful toward others and may come to view people as obstacles or interruptions to gaming. The user will regularly take "shortcuts" (i.e., actions that require the lowest effort) to continue or maintain their gaming. The prioritization of gaming develops a pattern of behavioral avoidance (e.g., school truancy, "sick days" from work, and avoiding social meals), not necessarily due to anxieties although they may also be present, but primarily with the intention of limiting the amount of time spent away from the gaming device. When unable to play, the user experiences distress or anhedonia, experiencing a loss or deprivation of purpose.

With the growing scientific interest in the broad effects of digital technology on users, there is a need for further studies that can clearly articulate the types and severity of harms experienced by individuals with IGD (Przybylski, Weinstein, \& Murayama, 2016). Given the above descriptions and examples, researchers should also consider: How does harm manifest differently according to certain groups, such as adolescents or individuals with comorbid conditions? Recent models of IGD and Internet-related disorders suggest that many of the behavioral and cognitive changes that produce harmful outcomes can be understood as stemming from the functional brain changes that occur in the context of IGD (Brand, Young, Laier, Wölfling, \& Potenza, 2016; Dong \& Potenza, 2014). Reduced self-regulation due to altered brain states leads to the prioritization of gamingrelated stimulation that develops a highly time-consuming and inflexible gaming routine that significantly interferes with normal functioning, including severe neglect of oneself, other people, and the real world.

A logical next step for research, and drawing insights from recent developments in gambling research (e.g., Browne et al., 2016), is to examine the way in which IGD harms may occur at different levels and degrees and how these might be assessed. At what point does the cost or harm of the gaming activity come to exceed its benefits? Many of these harms may be relatively minor in isolation (e.g., loss of some sleep), but harmful when they accumulate. Such work has important implications for enhancing our identification and understanding of individuals whose behavior is suspected to meet the definition of the proposed IGD criteria.

Funding sources: This work received financial support from a Discovery Early Career Researcher Award (DECRA) DE170101198 funded by the Australian Research Council.

Authors' contribution: DLK wrote the first draft of the paper and both authors contributed to and have approved the final manuscript.

Conflict of interest: The authors report no conflict of interest. They alone are responsible for the content and writing of the paper.

Ethics: Not applicable.

\section{REFERENCES}

American Psychiatric Association. (2013). Diagnostic and statistical manual of mental disorders (DSM-5) (5th ed.). Washington, DC: American Psychiatric Association. doi:10. 1176/appi.books.9780890425596

Baggio, S., Dupuis, M., Studer, J., Spilka, S., Daeppen, J. B., Simon, O., Berchtold, A., \& Gmel, G. (2016). Reframing video gaming and Internet use addiction: Empirical cross-national comparison of heavy use over time and addiction scales among young users. Addiction, 111(3), 513-522. doi:10.1111/add. 13192

Brand, M., Young, K. S., Laier, C., Wölfling, K., \& Potenza, M. N. (2016). Integrating psychological and neurobiological 
considerations regarding the development and maintenance of specific Internet-use disorders: An interaction of PersonAffect-Cognition-Execution (I-PACE) model. Neuroscience and Biobehavioral Reviews, 71, 252-266. doi:10.1016/j. neubiorev.2016.08.033

Browne, M., Langham, E., Rawat, V., Greer, N., Li, E., Rose, J., Rockloff, M., Donaldson, P., Thorne, H., Goodwin, B., Bryden, G., \& Best, T. (2016). Assessing gambling-related harm in Victoria: A public health perspective. Melbourne, Australia: Victorian Responsible Gambling Foundation.

Chuang, Y. C. (2006). Massively multiplayer online role-playing game-induced seizures: A neglected health problem in Internet addiction. CyberPsychology \& Behavior, 9(4), 451-456. doi:10.1089/cpb.2006.9.451

Dong, G., \& Potenza, M. N. (2014). A cognitive-behavioral model of Internet gaming disorder: Theoretical underpinnings and clinical implications. Journal of Psychiatric Research, 58, 7-11. doi:10.1016/j.jpsychires.2014.07.005

Dong, G., Wang, L., Du, X., \& Potenza, M. N. (2017). Gaming increases craving to gaming-related stimuli in individuals with Internet gaming disorder. Biological Psychiatry: Cognitive Neuroscience and Neuroimaging, 2, 404-412. doi:10.1016/j. bpsc.2017.01.002

Kaptsis, D., King, D. L., Delfabbro, P. H., \& Gradisar, M. (2016). Withdrawal symptoms in Internet gaming disorder: A systematic review. Clinical Psychology Review, 43, 58-66. doi:10. 1016/j.cpr.2015.11.006

King, D. L., \& Delfabbro, P. H. (2016). The cognitive psychopathology of Internet gaming disorder in adolescence. Journal of Abnormal Child Psychology, 44(8), 1635-1645. doi:10.1007/ s10802-016-0135-y

King, D. L., Kaptsis, D., Delfabbro, P. H., \& Gradisar, M. (2016). Craving for Internet games? Withdrawal symptoms from an 84-h abstinence from massively multiplayer online gaming. Computers in Human Behavior, 62, 488-494. doi:10.1016/j. chb.2016.04.020
Langham, E., Thorne, H., Browne, M., Donaldson, P., Rose, J., \& Rockloff, M. (2015). Understanding gambling related harm: A proposed definition, conceptual framework, and taxonomy of harms. BMC Public Health, 16(1), 80. doi:10.1186/s12889016-2747-0

Macgregor, D. M. (2000). Nintendonitis? A case report of repetitive strain injury in a child as a result of playing computer games. Scottish Medical Journal, 45(5), 150. doi:10.1177/ 003693300004500507

Männikkö, N., Billieux, J., \& Kääriäinen, M. (2015). Problematic digital gaming behavior and its relation to the psychological, social and physical health of Finnish adolescents and young adults. Journal of Behavioral Addictions, 4(4), 281-288. doi:10.1556/2006.4.2015.040

Przybylski, A. K., Weinstein, N., \& Murayama, K. (2016). Internet gaming disorder: Investigating the clinical relevance of a new phenomenon. American Journal of Psychiatry, 174(3), 230-236. doi:10.1176/appi.ajp.2016.16020224

Schüll, N. D. (2012). Addiction by design: Machine gambling in Las Vegas. Princeton, NJ: Princeton University Press.

Soroush, M., Hancock, M., \& Bonns, V. K. (2014, October). Selfcontrol in casual games: The relationship between Candy Crush Saga ${ }^{\mathrm{TM}}$ players' in-app purchases and self-control. In Games Media Entertainment (GEM), 2014 IEEE. doi:10. 1109/GEM.2014.7048099. Retrieved from https://touchlab. uwaterloo.ca/testing/wordpress/wp-content/uploads/ieee-gem2014 submission_56.pdf

World Health Organization [WHO]. (2014). Global status report on alcohol and health, 2014. Geneva, Switzerland: World Health Organization.

World Health Organization [WHO]. (2018). Gaming disorder. Retrieved from http://www.who.int/features/qa/gaming-disorder/en/

Wu, C. Y., Lee, M. B., Liao, S. C., \& Chang, L. R. (2015). Risk factors of Internet addiction among Internet users: An online questionnaire survey. PLoS One, 10(10), e0137506. doi:10. 1371/journal.pone.0137506 